\title{
The shallow processing of logical negation
}

\section{Guillermo Macbeth $^{1,2}$, Eugenia Razumiejczyk ${ }^{1,2}$, Maria del Carmen Crivello ${ }^{1}$, Mauro Fioramonti ${ }^{1}$, Carolina I. Pereyra Girardi ${ }^{2,3}$}

\author{
${ }^{1}$ National University of Entre Rios, Parana, Argentina \\ ${ }^{2}$ National Scientific and Technical Research Council, Argentina \\ ${ }^{3}$ University of Buenos Aires, Buenos Aires, Argentina
}

\section{Email address:}

g.macbeth@conicet.gov.ar (G. Macbeth), eugeniaraz@hotmail.com (E. Razumiejczyk), mcrivello@hotmail.com (M. del C. Crivello), mauro.fdd@gmail.com (M. Fioramonti), cpereyragirardi@gmail.com (C. I. P. Girardi)

\section{To cite this article:}

Guillermo Macbeth, Eugenia Razumiejczyk, Maria del Carmen Crivello, Mauro Fioramonti, Carolina I. Pereyra Girardi. The Shallow Processing of Logical Negation. Psychology and Behavioral Sciences. Vol. 2, No. 5, 2013, pp. 196-201.

doi: $10.11648 /$ j.pbs.20130205.15

\begin{abstract}
The aim of this study is to introduce a novel reasoning phenomenon concerned with the shallow processing of negation in the context of sentential reasoning. By analogy to other psychological explanations that account for superficial responses with conditionals, this study proposes an account for biconditionals derived from a recent theory of negation. This theory predicts that the psychological use of negation returns small scope products. This would happen because the human mind tends to avoid the working memory overload by simplifying its reasoning processes. A within-subjects experimental design was applied to test this conjecture. Results were consistent with such small scope negation prediction. The obtained evidence extends the observation of shallow reasoning processes to the negation of conjunctions and disjunctions that take the form of biconditionals. The results of this study support a mental models approach to account for the psychology of logical negation.
\end{abstract}

Keywords: Reasoning, Logic, Negation, Cognition, Shallow Processing

\section{Introduction}

Deductive reasoning is hard for humans [1]. Even simple deductive tasks often yield low performances [2]. This would probably happen because the human mind does not seem to work according to the rules of logic [3]. The psychological processing of deduction, in contrast, would proceed by representing mental simulations inferred from given information [1]. An important collection of evidence suggests that humans construct mental models to achieve deductive conclusions [4]. A mental model can be defined as an iconic representation of the world that restricts the working memory load to a manageable minimum [5]. This would happen by abstraction and combination of true statements that are derived from given information. A further comparison between such statements would yield the sought conclusion to a deductive problem or situation. This view is proposed by the Mental Models Theory (MMT) [1]. The MMT emphasizes the semantic and pragmatic variables of reasoning, whereas previous theories were focused on syntactic or formal components of human thought [4]. Semantic variables are concerned with meaning and pragmatic variables are concerned with the context in which such meaning takes place. Syntactic components are those related to abstract structures and to their formal properties. The MMT defines working memory as the short-term memory system that regulates reasoning [1]. This system would be responsible for the representation and inference of deductive processes [4].

The MMT and other reasoning theories have identified a collection of experimental phenomena that are of central interest for this study. These phenomena can be broadly described as products of a shallow mental processing. The term shallow in this paper refers to a weak semantic interaction between the new information entered in the cognitive system and previous knowledge. In the reasoning literature, Rips [6] took the expression shallow processing to label heuristic theories of syllogisms and to distinguish them from other approaches like the analytic theories or comprehension theories [7]. The shallow processing theories of reasoning are opposed to the deduction system hypothesis proposed by Rips [6]. According to the latter, deduction in humans work like a general purpose programming system, 
that is, an algorithm that applies a finite set of rules [8].

Three theories that account for shallow processes in reasoning were selected for this study to elaborate new predictions concerned with logical negation: the matching bias, the min-heuristic, and the atmosphere effect.

The matching bias occurs when the Wason's Selection Task (WST) produce responses critically anchored to features that are mentioned in the task instructions [9]. The WST is an experimental reasoning task that calls for the selection of evidence consistent with a given rule using four cards that include specific information in both sides. Experimental participants have to select which card or cards have to be known by both sides to evaluate the given rule. For example, a rule is given: if there is a $\mathrm{P}$ on one side of a card, then there is a 6 on the other side of the card. Then, subjects are presented four several cards that show a $P$, a 6 , and alternatives like a $\mathrm{Q}$, and a 5. The task is to decide which card or which cards have to be known by both sides to prove that the rule is true for the given set of cards. Elaborated inferential responses are expected in the WST, but it was observed instead that subjects perform a rapid response that matches superficial features mentioned in the rule [10]. If $P$ was mentioned in the rule, then subjects' overall responses select the $\mathrm{P}$ card. Instead of thinking in terms of the conditional as logic operator, subjects match the response with given superficial information. An extensive analysis of several matching bias variants has been proposed by Evans [9].

The min-heuristic account has been introduced in the context of the Probability Heuristics Model or PHM proposed by Chater \& Oaksford [11]. The PHM is an explanation that deals with quantifiers in the context of syllogistic inference. The min-heuristic account predicts that conclusions should take the scope of the least informative quantifier presented in the premises of a given syllogism. Chater \& Oaksford [11] proposed that a minimal mental processing is expected when this heuristic is activated.

The atmosphere effect accounts for a typical conservative response modality that preserves superficial features in the context of syllogistic inference [12]. The atmosphere effect occurs when the quantifiers presented in the premises promote conclusions structured by the same quantifiers. This phenomenon was first identified by Sells [13], but further research has found several variants [1]. The atmosphere effect can be understood in a broad sense as a sort of shallow persistence anchored to given information in syllogistic tasks.

The general aim of this paper is to introduce a new superficial processing phenomenon concerned with logical negation. This new shallow phenomenon can be linked in a broad sense to the matching bias, the min-heuristic, and the atmosphere effect. Although these three previous phenomena deal with conditionals in syllogistic inference, the present study proposes that a shallow processing also occurs with biconditionals in sentential reasoning.

This paper is organized as follows. First, we introduce logical and psychological concepts that are relevant for the phenomenon of interest. After introducing definitions for sentences and connectives, two particular logical equivalences known as DeMorgan's laws are described. These laws are concerned with negation and take the form of biconditionals. Then, a recent theory of negation is commented and postulated as relevant framework for the phenomenon of interest. Finally, we present and discuss an experiment that generated evidence concerning the shallow processing of DeMorgan's laws in the context of the MMT.

\section{Sentential Reasoning}

Sentential reasoning is a subfield in the deduction research, specifically concerned with the mental processing of sentences containing connectives such as and, or, and not, among others $[1,14]$.

\subsection{Sentences and Connectives}

According to mathematical logic [15], a proposition or sentence is an expression that can be considered true or false but not both. If a truth value cannot be attributed to an expression, then such expression is not a sentence. For example, a question is not a sentence, even if it makes sense in a given language. A sentence can be represented in an abstract manner by letters. For example, the sentence "London is a city" can be represented by the letter $p$. Similarly, the sentence "Europe is a continent" can be expressed by the letter $q$. Sentences can be combined through connectives, which are abstract functions expressed through words like and, and or. These words operate the function of conjunction and disjunction, respectively. An example of conjunction would be "London is a city and Europe is a continent", which can be expressed as $p$ and $q$. Another important connective is expressed through the term if..., then..., which is defined as the conditional operator.

The disjunction connective has two variants, the inclusive and the exclusive. The inclusive disjunction considers that a disjunctive sentence, e.g. $p$ or $q$ is true when $p$ is true, or when $q$ is true, or when both $p$ and $q$ are simultaneously true. The exclusive disjunction is true when either $p$ is true or when $q$ is true, but is false when both $p$ and $q$ are simultaneously true.

Sentences can be further classified as atomic or molecular [16]. An atomic sentence is one without connectives and without negation. A molecular sentence is one that has connectives linking two or more atomic sentences, or is a denied atomic sentence.

Mathematical logic further states that two sentences are equivalent when they are syntactically interchangeable. Two equivalent sentences express exactly the same idea, without information lost or redundancy. Equivalences take the form of a biconditional, that is, a relation between two structures that mutually imply. If $p$ implies $q$, and $q$ implies $p$, then $p$ and $\mathrm{q}$ are equivalent. This specific relation is defined as biconditional [15]. The equivalence in logic is 
analogous to the equation in mathematics.

A particularly important operation for sentential reasoning is negation. Given the sentence "London is a city", its negation is another sentence that can be expressed as, e.g., "London is not a city" or "It is not true that London is a city". Given $p$, negation returns not $p$. Negations can operate on atomic sentences, but also on molecular sentences. The latter has been proved to be extremely difficult for experimental participants with no training in logic or mathematics [17]. This paper is focused on the mental processing of one of these cases, i.e., the negation of conjunctions and disjunctions. Such structures are known in logic as DeMorgan's laws.

\subsection{DeMorgan's Laws}

It can be formally proved that the negation of a conjunction is equivalent to an inclusive disjunction, and the negation of an inclusive disjunction is equivalent to a conjunction. A mathematical proof for both equivalences is available in [18]. These equivalences are valid in natural language, which can be broadly defined as the ordinary language that we employ everyday. English and Spanish are examples of natural language. Application examples of these equivalences are presented as follows. The sentence "It is not true that: London is a city and Europe is a continent" is equivalent to the sentence "London is not a city, or Europe is not a continent, or both". Conversely, the sentence "It is not true that: London is a city, or Europe is a continent, or both" is equivalent to "London is not a city and Europe is not a continent". The formulation of these equivalences as logical laws is often attributed to the British mathematician Augustus DeMorgan [19], who lived and made many important contributions to logic and mathematics during the 19th century. Nevertheless, this attribution is erroneous because these equivalences were well known by medieval logicians like Walter Burleigh, who wrote his Tractatus De Consequentiis in 1302 [20].

In sum, DeMorgan's law 1 state that sentences of the form not ( $p$ and $q$ ) are equivalent to the corresponding sentence of the form (not p) or (not q), or both. DeMorgan's law 2 state that sentences of the form not ( $p$ or $q$, or both) are equivalent to the corresponding sentence of the form (not $p$ ) and (not $q$ ).

\subsection{A Theory of Negation}

An extension of the MMT concerned with negation has been recently proposed by Khemlani, Orenes, and Johnson-Laird [17]. This semantic-driven theory covers the meaning, representation, and use of negation. Its core prediction postulates that negation is a mental process that takes a single argument referred to a single set of possibilities and returns the complement of that set. This psychological definition replicates in the context of the MMT the mathematical theory of sets. A complementary set in mathematical set theory can be defined through an abstract function that operates on a given set and returns a new set formed by the elements that are not included in the first set [16]. Complementation in set theory is analogous to negation in sentential reasoning. The latter can be considered as a particular case of the former.

The MMT of negation postulates five psychological predictions, from which the first one has critical importance for this study. Khemlani et al [17] posits that negation yields a small scope result. If negation operates on a molecular sentence, it is predicted that experimental participants would generate responses structured as partial negations limited to the corresponding atomic sentences. This would happen because a small scope negation demands lower mental computations. A corollary for this prediction would be that DeMorgan's laws should not be successfully processed by participants with no training in logic or mathematics. More specifically, if participants are asked to negate a conjunction or a disjunction and to find an equivalent sentence, the most frequent responses should be structured as small scope negations. For DeMorgan's law 1, composed by sentences of the form not ( $p$ and $q$ ), the predicted response would be (not $p$ ) and (not $q$ ) instead of the correct response (not p) or (not q), or both. For DeMorgan's law 2, composed by sentences of the form not ( $p$ or $q$, or both), the predicted response would be (not $p$ ) or (not q) instead of the correct response (not p) and (not q). According to Khemlani et al [17], this would happen because a small scope negation demands a lower working memory load than a full scope negation. These authors further argue that this psychological process is activated heuristically, that is, as an automatic cognitive tool that operates in a fast and frugal manner [21].

\section{Method}

A within-subjects experimental design was applied to test the small scope hypothesis derived from the MMT of negation [17]. The particular case of DeMorgan's laws was selected to construct the materials and formulate the experimental hypotheses.

\subsection{Participants}

A random sample of 86 undergraduate students of social sciences was recruited at a public university located in the city of Parana, Argentina. All the participants had no formal education in logic or mathematics. The average age was 24.55 years old $(S D=4.02)$. Female participants $(n=71)$ represent $82.6 \%$ of the sample.

\subsection{Materials and Procedure}

An experimental task with DeMorgan's laws in a selection paradigm was constructed. Table 1 presents an example of the experimental task. Instructions asked participants to find the small letters sentence that was equivalent to the capital letters sentence. The experiment was administered in paper and pencil at the beginning of a regular class. All the participants completed an informed 
consent before starting the experiment. The experimental sessions lasted between ten and fifteen minutes.

In a selection paradigm the task requires to answer by selecting one response from a list of several options. The selection paradigm is the alternative strategy to the construction paradigm, which requires participants to build their own responses instead of selecting it from a given list of options. The experimental task constructed for this experiment included 8 items with 4 response options. Each item first presented the negation of a conjunction or the negation of a disjunction. Then, the participant was asked to find an equivalent sentence for the given negation among a list of possible responses. The negation sentence was presented in capital letters, and the response options sentences were presented in small letters. Instructions further remarked that two sentences were equivalent when they had the same abstract meaning. Finally, instructions mentioned that only one of the four response options was the correct response.

Table 1. Experimental task example.

IT IS NOT TRUE THAT: LONDON IS A CITY AND EUROPE IS A CONTINENT

a) London is not a city and Europe is not a continent. *

b) London is not a city or Europe is not a continent, or both. **

c) If London is not a city, then Europe is not a continent.

d) London is not a city or else Europe is not a continent.

Note: one asterisk indicates the small scope negation response, which would be the product of a shallow processing. Two asterisks indicate the correct response. This item applies DeMorgan's law 1.

The response options for law 1 were: a conditional (see option c in Table 1), a conjunction (option a), an exclusive disjunction (option d), and the correct answer (inclusive disjunction, option $b$ ). The response options for law 2 were: a conditional, an exclusive disjunction, an inclusive disjunction, and the correct answer (conjunction). The atomic sentences linked by these connectives were denied in all cases. The sequence of response options within each item and the general sequence of items were both randomized. The conditional was included as a response option because mathematical logic states that a disjunction can be transformed into a conditional [16]. Additionally, a conjunction can be transformed into a disjunction (applying DeMorgan's laws), which justifies the inclusion of the other response options.

An indexes construction strategy was applied to compare all the response options. The Correct-Index was defined as the sum of equivalences selection for the corresponding DeMorgan's law. The Transformation-Index was the sum of erroneous conditional responses. The Scope-Index was the sum of erroneous exclusive disjunction responses. Finally, the Surface-Index was defined as the sum of small scope negation responses, that is, (not $p$ ) and (not $q$ ) for law 1 and (not $p$ ) or (not $q$ ) for law 2. These Indexes were defined as vectors obtained through the sum of the corresponding responses given by each experimental participant to the eight items of the task. Indexes for law 1 (four items) were calculated separately from indexes for law 2 (four items).

\subsection{Hypotheses}

Hypothesis $\mathrm{H} 1$ predicts that the most frequent response to DeMorgan's law 1 items should be the one captured by the Surface-Index, that is (not p) and (not q). Hypothesis $\mathrm{H} 2$ predicts for law 2 that the most frequent response should be (not p) or (not q), which is captured by the corresponding Surface-Index. Both hypotheses are derived from the negation theory of the MMT and justified by analogy to the shallow processing observed in other reasoning phenomena like the matching bias, the min-heuristic and the atmosphere effect. Hypotheses H1 and $\mathrm{H} 2$ are predictions based on the small scope negation conjecture proposed by Khemlani et al [17].

\subsection{Results and Discussion}

No significant differences were found between male and female participants after Mann-Whitney $U$ test for the Transformation-Index $(z=-1.234, p=.217$, $\mid$ Cliff's Delta $\mid$ $=.167)$, the Scope-Index $\left(z=-0.499, p=.618, \mid\right.$ Cliff $^{\prime} s$ Delta $\mid=.07)$, the Correct-Index for law $1(z=-1.345, p$ $=.179, \mid$ Cliff's Delta $\left.^{\prime}=.156\right)$, the Correct-Index for law 2 $(z=-1.114, p=.265, \mid$ Cliff's Delta $\mid=.172)$, and the Surface-Index $(z=-0.53, p=.596, \mid$ Cliff's Delta $\mid=.086)$. The absence of difference between male and female participants in this DeMorgan's reasoning task is consistent with previous studies on verbal reasoning [22].

Both experimental hypotheses, $\mathrm{H} 1$ and $\mathrm{H} 2$, resulted consistent with the evidence. The overall most frequent response was the small scope negation captured by the Surface-Index for both laws. Tables 2 and 3 present descriptive statistics of each index for law 1 and low 2, respectively.

Table 2. Descriptive statistics of indexes for law 1

\begin{tabular}{lcc}
\hline Index & Mean & Standard Deviation \\
\hline Surface-Index & 2.99 & 1.30 \\
\hline Transformation-Index & 0.43 & 0.79 \\
\hline Scope-Index & 0.34 & 0.67 \\
\hline Correct-Index & 0.24 & 0.53 \\
\hline
\end{tabular}

Table 3. Descriptive statistics of indexes for law 2

\begin{tabular}{lcc}
\hline Index & Mean & Standard Deviation \\
\hline Surface-Index & 2.23 & 1.50 \\
Correct-Index & 1.06 & 1.23 \\
Scope-Index & 0.49 & 0.86 \\
Transformation-Index & 0.22 & 0.58 \\
\hline
\end{tabular}

Tables 4 and 5 present statistical comparisons between the Surface-Index and the other indexes for law 1 and law 2, respectively. The Sign test was applied in all cases because some vectors resulted incompatible with the normality and 
homogeneity of variances assumption required to perform parametric comparisons. A non-parametric effect size was further calculated. The Cliff's Delta effect size was chosen. For this effect size, the absolute value was informed because the plus or minus polarities depend only on which vector was entered first to the algorithm [23]. The smallest effect size is associated to zero, whereas the biggest effect size is associated to one for the Cliff's Delta absolute value.

Table 4. Comparison between Surface-Index and other indexes for law 1

\begin{tabular}{cccc}
\hline & Trans-Index & Scope-Index & Correct-Index \\
\hline Hypothesis & Surf $>$ Trans & Surf $>$ Scope & Surf $>$ Correct \\
$z$ & -7.400 & -7.444 & -7.938 \\
$p$-value & $<.001$ & $<.001$ & $<.001$ \\
$\mid$ Cliff's $\delta \mid$ & .844 & .863 & .883 \\
Effect size & large & Large & large \\
\hline
\end{tabular}

Note: Trans means Transformation-Index, Surf means Surface-Index, Scope means Scope-Index, Correct means Correct-Index. All the comparisons are between the Surface-Index and the index indicated in each column.

Table 5. Comparison between Surface-Index and other indexes for law 2

\begin{tabular}{cccc}
\hline & Correct-Index & Scope-Index & Trans-Index \\
\hline Hypothesis & Surf $>$ Correct & Surf $>$ Scope & Surface $>$ Trans \\
$z$ & -3.327 & -5.539 & -6.697 \\
$p$-value & $<.001$ & $<.001$ & $<.001$ \\
$\mid$ Cliff's $\delta \mid$ & .441 & .648 & .746 \\
Effect size & medium & large & large \\
\hline
\end{tabular}

Note: see note on Table 4.

The distance between the Surface-Index and the other indexes resulted medium to large. This result suggests that the overall response to the proposed reasoning experiment can be explained by a shallow processing. A weak semantic elaboration might have happened. DeMorgan's laws received a small scope negation processing, as predicted by Khemlani et al [17] in the context of the MMT.

The effect size between the Surface-Index and the Correct-Index resulted large for law 1 and medium for law 2. This asymmetry is also predicted by the MMT of negation. Such phenomenon would happen because law 2 (a conjunction) demands a lower working memory load than law 1 (an inclusive disjunction). A recent study conducted by Khemlani, Orenes, and Johnson-Laird [24] contributed novel evidence for this asymmetric phenomenon for the particular case of DeMorgan's laws.

\section{Conclusions}

Research on reasoning has identified several phenomena that can be described as products of a shallow mental processing. Three of them are explained through the matching bias [9, 10], the min-heuristic [11], and the atmosphere effect $[12,13]$. These particular cases are concerned with syllogisms and conditionals. One common element between the three is the weak semantic processing of deductive information. This paper aims to introduce a novel phenomenon in which the same shallow processing occurs for the specific case of DeMorgan's laws cognition. An analogous shallow processing would occur for biconditionals concerned with negations. The theory of negation formulated within the MMT predicted this result. The small scope negation would be produced by the spontaneous tendency to avoid working memory overload. It seems that the human mind prefers to spare resources insofar the environmental adjustment is achieved [1]. A processing heuristic described by Khemlani et al [17] as small scope negation resulted consistent with the experimental results of this study. This would probably happen because responses structured according to superficial features seem to be less cognitive demanding than a deep re-structuring of sentences containing negations of other connectives like conjunctions and disjunctions.

One limitation of this study is concerned with the contextual variables neglect. Replications of these hypotheses testing in naturalistic contexts are recommended. Previous findings suggest that adequate ecological designs increase the correct responses frequency [21]. Another limitation is concerned with the lack of response time measures. A stronger test for the novel phenomenon proposed in this paper should obtain shorter latencies for the Surface-Index when compared to any other index. This would happen if responses captured by such other indexes are directly neglected by participants. This result would be consistent with the small scope negation conjecture.

In sum, this paper introduced a novel phenomenon of shallow processing in reasoning tasks concerned with logical negation. Previous phenomena were concerned with conditionals, whereas the present study contributes evidence concerning biconditionals for the particular case of DeMorgan's laws.

\section{Acknowledgements}

Authors thank Sangeet Khemlani for his helpful comments on the direct evaluation of DeMorgan's laws proposed in this study and Guillermo Moro for the English grammar review.

\section{References}

[1] P. N. Johnson-Laird, How we reason, Oxford, UK: Oxford University Press, 2008.

[2] P. C. Wason, and P. N. Johnson-Laird, Psychology of reasoning, Cambridge, MA: Harvard University Press, 1972.

[3] P. N. Johnson-Laird, "Against logical form," Psychologia Belgica, vol. 50, pp. 193-221, 2010.

[4] P. N. Johnson-Laird, "Mental models and human reasoning," Proceedings of the National Academy of Sciences, vol. 107, pp. 18243-18250, 2010. 
[5] P. N. Johnson-Laird, and R. M. J. Byrne, Deduction, Hillsdale, NJ: Lawrence Erlbaum Associates, 1991.

[6] L. J. Rips, "Deduction," in The psychology of human thought, R. J. Sternberg, and E. E. Smith (Eds.), Cambridge, UK: Cambridge University Press, 1988, pp. 116-152.

[7] W. Schroyens, W. Schaeken, W. Fias, and G. d'Ydewalle, "Heuristic and analytic processes in propositional reasoning with negatives," Journal of Experimental Psychology: Learning, Memory, and Cognition, vol. 26, pp. 1713-1734, 2000.

[8] L. J. Rips, The psychology of proof, Cambridge, MA: MIT Press, 1994.

[9] J. S. B. T. Evans, "Matching bias in conditional reasoning: Do we understand it after 25 years?," Thinking \& Reasoning, vol. 4, pp. 45-110, 1998.

[10] E. J. N. Stupple, L. J. Ball, and D. Ellis, "Matching bias in syllogistic reasoning: Evidence for a dual-process account from response times and confidence ratings," Thinking \& Reasoning, vol. 19, pp. 54-77, 2013.

[11] N. Chater, and M. Oaksford, "The probability heuristics model of syllogistic reasoning," Cognitive Psychology, vol. 38, pp. 191-258, 1999.

[12] N. E. Wetherick, and G. J. Gilhooly, "Atmosphere, matching, and logic in syllogistic reasoning," Current Psychology, vol. 14, pp. 169-178, 1995.

[13] S. B. Sells, "The atmosphere effect: an experimental study of reasoning," Archives of Psychology, vol. 29, pp. 3-72, 1936.

[14] F. J. Springston, and H. H. Clark, "And and or, or the comprehension of pseudoimperatives," Journal of Verbal Learning and Verbal Behavior, vol. 12, pp. 258-272, 1973.
[15] P. Suppes, and S. Hill, First course in mathematical logic, Mineola, NY: Dover Publications, 1992.

[16] R. Garnier, and J. Taylor, 100\% mathematical proof, Chichester, UK: John Wiley \& Sons, 1996.

[17] S. Khemlani, S. Orenes, and P. N. Johnson-Laird, "Negation: A theory of its meaning, representation, and use," Journal of Cognitive Psychology, vol. 24, pp. 541-559, 2012.

[18] G. Macbeth, E. Razumiejczyk, and G. Campitelli, "The abstraction effect on logic rules application," Education Sciences and Psychology, vol. 24, pp. 85-96, 2013.

[19] A. DeMorgan, Formal logic or the calculus of inference necessary and probable, London, UK: Taylor \& Walton, 1847.

[20] P. Boehner, Medieval logic, Manchester, UK: Manchester University Press, 1952.

[21] G. Gigerenzer, P. M. Todd, and the ABC Research Group, Simple heuristics that make us smart, New York, NY: Oxford University Press, 1999.

[22] R. Colom, M. J. Contreras, I. Arend, O. García Leal, and J. Santacreu, "Sex differences in verbal reasoning are mediated by sex differences in spatial ability," The Psychological Record, vol. 54, pp. 365-372, 2004.

[23] G. Macbeth, E. Razumiejczyk, and R. D. Ledesma, "Cliff's Delta Calculator: A non-parametric effect size program for two groups of observations," Universitas Psychologica, vol. 10 , pp. 545-555, 2011.

[24] S. Khemlani, I. Orenes, and P. N. Johnson-Laird, "Negating compound sentences," in Building bridges across cognitive sciences around the world, N. Miyake, D. Peebles, and R. P. Cooper (Eds.), Austin, TX: Cognitive Science Society, 2012, pp. 575-580. 